\title{
General Situation of Research on Treatment of Insomnia by Different Scalp Acupuncture Schools
}

\author{
Xin Han', Yuanyuan $\operatorname{Ren}^{2}$ ** \\ ${ }^{1}$ Shaanxi University of Traditional Chinese Medicine, Xianyang 712046, China \\ ${ }^{2}$ Xi' an Traditional Chinese Medicine Hospital, Xi'an 710000, China \\ *Correspondence Author
}

\begin{abstract}
It is well known that scalp acupuncture has considerable efficacy in treating insomnia, and many scalp acupuncture schools have their own characteristics. Therefore, the development of scalp acupuncture in the treatment of insomnia also requires in-depth research on the scalp acupuncture genre to develop the clinical advantages of each genre. Therefore, this study aims to organize the literature related to the treatment of insomnia by scalp acupuncture, in order to provide the best stimulation method for clinical scalp acupuncture therapy to treat insomnia, reduce the blindness of clinical scalp acupuncture selection, and promote the innovation of acupuncture and moxibustion knowledge and scalp acupuncture genre. Provide information for further development.
\end{abstract}

Keywords: Scalp acupuncture school, Insomnia, Treatment.

\section{Introduction}

Scalp acupuncture refers to a method of using acupuncture to stimulate the acupoints or treatment areas of the cerebral cortex in the corresponding projection area of the scalp to treat and prevent diseases, referred to as scalp acupuncture or scalp acupuncture[1]. Scalp acupuncture is derived from the "Huangdi Neijing", "Lingshu-Five Chaos" says: "The head is heavy and dizzy...those who are on the head, take the Tianzhu and Dazhu". Studies have found that scalp acupuncture is effective in treating brain-derived diseases such as insomnia, epilepsy, depression, and stroke[2,3]. Since the 1950s, the clinical application of scalp acupuncture has attracted the attention of doctors. After years of continuous exploration, many schools of scalp acupuncture have been formed. The continuous development of each school has greatly promoted modern acupuncture and moxibustion, scalp acupuncture theory and clinical practice. Practice development. Insomnia is a disease characterized by difficulty in falling asleep and difficulty in maintaining sleep. The main symptoms of long-term inability to obtain normal sleep are[4]. Insomnia seriously affects the quality of life and mental state of patients. Long-term insomnia can easily lead to mental disorders and endocrine disorders, and increase the risk of other diseases, such as obesity, anxiety, depression, and hypertension. However, western medicine has certain side effects. Cognitive therapy reduces the efficacy due to cost and patient compliance issues. Studies[3-6] have pointed out that scalp acupuncture has a good effect in the treatment of insomnia. Therefore, sort out the literature related to the treatment of insomnia by scalp acupuncture, compare the theoretical basis and point selection of the currently widely used scalp acupuncture school, to provide the best stimulation method for the clinical scalp acupuncture treatment of insomnia, and reduce the choice of clinical scalp acupuncture. Blind research is necessary.

2. The Theoretical Basis and Selected Areas (Acupoints) of Various Schools in the Treatment of Insomnia

\subsection{Jiao's Scalp Acupuncture}

Jiao's scalp acupuncture combines the theory of modern medical cerebral cortex function positioning, and divides the head into 19 stimulation areas such as mental and emotional area, vasomotor area, and dizziness area. Acupuncture can treat the corresponding area. Disease syndrome[7]. Studies have found that acupuncture at the fainting area can alleviate affective and mental disorders caused by hippocampal damage, and reduce the possibility of insomnia; acupuncture at the vasomotor area and mental-emotional area can promote the inhibitory effect of neurotransmitters on neurons, and reduce The excitability of the body achieves the effect of regulating emotions, reducing the mental pressure and psychological burden caused by insomnia[8,9]. It is clinically believed that Jiao's scalp acupuncture is more direct and comprehensive than traditional acupuncture for the treatment of liver depression and fire-type insomnia, which can improve symptoms and prognosis.

\subsection{Qin's Scalp Acupuncture}

Qin's scalp acupuncture is divided into 4 sections of the head according to the classification of headaches. It is believed that the injection method of Qin's flying needle three methods in the top area can be achieved by a combination of three needles. The effect of soothing the liver and regulating qi, promoting qi and dredging collaterals, can be used to treat insomnia of liver stagnation and qi stagnation. Ren Menglei et al.[10] believe that Baihui points have the functions of relaxing the meridians and activating the collaterals, nourishing the brain and strengthening the brain. The fontanelle can pass the bone sutures to the meridian and enter the brain orifices. Shenting is closely related to the expression of human emotions. All acupoints are located in the governor channel. Above, it can be used to treat insomnia of liver stagnation and qi stagnation. The results show that Qin's scalp acupuncture is more effective than estazolam, and can effectively improve the patient's sleep quality and day dysfunction.

\subsection{Zhu's Scalp Acupuncture}


Zhu's Scalp Acupuncture is based on the international standardization plan, with Baihui as the midpoint, and the Du Channel as the distribution zone dividing the treatment area on the midline. The scalp acupuncture area is simplified into 9 zones (frontal top). Belt, parieto-occipital belt, anterior temporal belt, anterior parietal knot, etc.)[11]. Zhu's scalp acupuncture believes that the forehead zone and the side 1 zone take the Du Channel as the midline, and Baihui divides yin and yang before and after. If acupuncture is carried out with breathing, yin and yang can be adjusted for the treatment of mental illness, neurosis, etc.[12]. Studies have shown that[13] Zhu's scalp acupuncture combined with body acupuncture is better than Guipi Decoction in improving the quality of life, sleep quality, and TCM syndrome score.

\subsection{Fang's Scalp Acupuncture}

Fang's scalp acupuncture roughly regards the human body's total motion system as a microcosm of lying on the head, and the human sensory center as a microcosm of the hair lying on the forehead. Dirty, dirty, inverted image 4 parts and 11 large centers. Fang's scalp acupuncture is often used in the treatment of insomnia, such as the Fuxian head, Fuzangshangjiao, thinking, signal, memory and other acupoints. By regulating the high-level activities of the cerebral cortex, it controls and regulates the activities of the whole body meridians, so as to achieve calmness, sleep, and mental sentiment. The role of[14]. An Junming et al., Dong Bo et al.[14,15] used acupuncture points such as thinking and signal to treat insomnia, and the total effective rate reached 95.8\% and $96.7 \%$, respectively. They all believed that Fang's scalp acupuncture can promote blood circulation in the head and improve nerve conduction function. Regulate neuromuscular excitability, suitable for the treatment of various types of insomnia.

\subsection{Lin's Scalp Acupuncture}

Lin's scalp acupuncture believes that stimulating specific areas of the head can improve the blood flow of the corresponding parts of the cerebral cortex, increase the number of neurotrophic factors in the brain, and thus prevent and treat brain-derived diseases. It is divided into five frontal needles, 6 areas including pre-exercise area and language formation area. In the treatment of insomnia, the main choice is the intellectual and emotional area (ie, the frontal five needles), the worry area, and the sensory area of the parietal lobe $1 / 5[16]$. The position of the frontal five needles belongs to the projection of the frontal joint area of the cerebral cortex on the scalp, and is closely related to intelligence and mental activity. If the disease affects the patient's intelligence, personality and spirit, etc. Studies have found that in the worry area (left upper frontal gyrus, middle frontal gyrus), the less brain cell activity there is, the more unstable the mood, which is closely related to emotional changes. Therefore, stimulating the area can treat symptoms such as insomnia[17]. Research[18] clinical comparative observation of the use of frontal five needles and body acupuncture confirmed that the frontal five has a definite effect on insomnia.

\subsection{Standardized Scalp Needles and Threads}

In the "International Program for Standardization of Scalp
Acupuncture Point Names"[19] formulated by the Chinese Society of Acupuncture and Moxibustion, there are a total of 14 standardized scalp needles in four parts of the frontal area, parietal area, temporal area, and occipital area. Clinically, scalp stitches in the forehead and apical areas are often used to treat insomnia. The governor channel enters the medullary collateral brain, and the frontal midline and top midline are on the governor channel line. After acupuncture, it can calm the liver and suppress the yang, calm the nerves. The frontal line 1 is on the foot sun bladder meridian, the frontal line 2 is on the foot shaoyang gall bladder meridian, and the frontal line 3 is mainly used for the treatment of hypojiao disease. Insomnia is closely related to visceral dysfunction. It is mostly caused by depression, thoughts and other excessive emotions, yin and yang Dysfunction is caused by the dysfunction of the viscera. After acupuncture, it mobilizes the meridian qi and blood of the Sun, Shaoyang, and Du Channel to go to the Rongnao orifice, submerging the yang into the yin, and can be used to treat insomnia[20].

\section{Manipulations for Treatment of Insomnia by Genre}

\subsection{Acupuncture Angle}

Lin's scalp needles are more flat needles, and straight needles can be selected at the positive reaction point[21], the others are more flat or oblique needles, and Fang's scalp needles require straight needles. Into the needle.

\subsection{Acupuncture Depth}

Fang's scalp acupuncture emphasizes that it must reach the periosteum. Lin's scalp acupuncture requires acupuncture to be close to the periosteum, and most of the others advocate puncturing under the cap-shaped aponeurosis.

\subsection{Acupuncture Method}

Most scalp acupuncture methods are consistent with traditional acupuncture, namely lifting and twisting, such as Tang's. On the basis of lifting and twisting, Fang's head needles are recommended to be pressed heavily. Yu's needles do not use the lifting method. Jiao's, Liu's, and Wang's needles are mostly twisted. Lin's head for the needle, choose the aspiration method for the needle, and for the Zhu's scalp, choose the air method for the needle[22-24].

\subsection{Needle Retention Time}

Jiao's and Yu's suggest that conventional needle retention is 30 minutes; Fang's, international standard scalp acupuncture, etc., choose 30 minutes or extend the needle retention time according to the condition; Lin's recommends that the needle be retained for more than 1 hour, and Yu's can $8-12$ hours, Zhu's needle can stay 2-72hours. Every scalp acupuncture school uses intermittent movement to retain the needle[25].

\section{Discuss}

Summarizing and analyzing the research on the treatment of insomnia by scalp acupuncture in various schools, it is found 
that scalp acupuncture should have an absolute advantage over single-target drugs in the treatment of insomnia. With the increase of clinical reports and the in-depth research of scalp acupuncture, the influence of scalp acupuncture is continuously increasing[26-30]. However, the effectiveness of scalp acupuncture in the treatment of insomnia is still controversial. The curative effect of acupuncture is directly related to the selection of acupoints and the effective amount of stimulation. The theoretical basis and positioning principles of each scalp acupuncture school are different, and even the same fixed acupoint area has different therapeutic effects in different schools. This has led to the need for acupuncture sites, acupuncture, and replenishing and reducing techniques in clinical operations. It's different. These contradictions and doubts will inevitably bring many difficulties to clinical medical workers, and then affect the further development of scalp acupuncture. It is difficult to form a unified and effective treatment plan for the treatment of insomnia by scalp acupuncture due to various acupuncture methods and personal tendency to select acupoints based on syndrome differentiation. This phenomenon should not only be reflected in the treatment of insomnia by scalp acupuncture. Therefore, We should strengthen clinical research on scalp acupuncture, find out the internal laws of scalp acupuncture diagnosis and treatment of diseases, continuously optimize the scalp acupuncture plan, find the best acupoint positioning, acupuncture techniques and quantitative standards, improve the theoretical and practical level of acupuncture practitioners, and further explore Its scientific connotation and therapeutic value can improve the effect of clinical insomnia treatment and promote the application and promotion of scalp acupuncture therapy.

\section{Acknowledgement}

This work is supported by the projects: 1) 2020-2022 subject project of Xi'an Science and Technology Bureau of Shaanxi Province (Project number: 20YXYJ001 (2)); and 2) 2021-2022 project of the Xi'an Municipal Health Commission, Shaanxi Province (project number: 2021yb03).

\section{References}

[1] Jia Chunsheng, Feng Shulan. Acupuncture and moxibustion[M]. Beijing: Science and Technology Press, 2017: 224

[2] Yan Haijun, Fu Haiyan. Research on the schools of contemporary Chinese medicine based on literature analysis[J]. Liaoning Journal of Traditional Chinese Medicine, 2017, 44(4): 720-722.

[3] Daley M, Morin CM, Leblanc M, et al. The economic burden of insomnia: Direct and indirect costs for individuals with insomnia syndrome, insomnia symptoms, and good sleepers[J]. Sleep, 2009, 32(1): 55-64.

[4] Xu Xiaoru, Wang Fuchun, Wang Zhihong. The effect of Sancai and Baihui points on sleep diary and TPF of patients with primary insomnia[J]. Chinese Journal of Traditional Chinese Medicine, 2018, 33(5): 1808-1811

[5] Jiang Hailin, Liu Chengyu, Wang Fuchun, et al. Observation on the clinical efficacy of electro acupuncture at a single point and compatible points in the treatment of primary insomnia[J]. Chinese Journal of Traditional Chinese Medicine, 2019, 34(5): 2266-2269

[6] Li Ying, Zhang Xiaoyang, Bao Fei, et al. Current status and analysis of clinical efficacy of acupuncture in the treatment of primary insomnia[J]. Chinese Acupuncture and Moxibustion, 2018, 38(7): 793-797

[7] Jiao Shunfa. Jiao Shunfa scalp needle[M]. Beijing: People's Medical Publishing House, 2009.

[8] Liu Zhenhua, Wang Shijun. The effect of acupuncture at Sishencong and Baihui on the expression of clock genes and amino acid neurotransmitters in brain tissue of insomnia rats[J]. Chinese Journal of Gerontology, 2015, 35(21): 6067-6069.

[9] Li Xinhao, Li Min, Wu Jiamin, et al. Clinical observation of Jiao's scalp acupuncture in the treatment of insomnia caused by liver depression and fire [J]. Herald of Chinese Medicine, 2018, 24(21): 93-94, 108.

[10] Ren Menglei, Qin Min, Zeng Xuexue. Observation on the clinical efficacy of Qin's scalp acupuncture combined with plum blossom needle tapping in the treatment of insomnia due to stagnation of liver and qi[J]. Journal of Acupuncture and Moxibustion, 2017, 33(12): $52-55$.

[11] Kong Yaoqi. A brief discussion on Zhu Mingqing's scalp acupuncture technique[J]. Journal of Traditional Chinese Medicine, 1988(7): 12-14.

[12] Chen Zhiyao, Min Jiahui, Huang Wenting, et al. Clinical application status and regular analysis of scalp acupuncture in the past 5 years[J]. Asia-Pacific Traditional Medicine, 2019, 15(01): 157-160.

[13] Chen Jiayao, Lao Jinxiong. Clinical study on Zhu's scalp acupuncture combined with body acupuncture in the treatment of insomnia with deficiency of the heart and spleen[J]. Journal of Acupuncture and Moxibustion, 2019, 35(11): 25-28.

[14] An Junming, Huang Linna, Dong Lianhe, etc. Shaanxi scalp acupuncture treatment of 24 cases of insomnia[J]. Chinese Acupuncture and Moxibustion, 2012, 32(12): 1093-1094

[15] Dong Bo, Huang Linna. Flying needle method to treat insomnia[J]. Journal of Clinical Acupuncture and Moxibustion, 2012, 28(8): 43-44

[16] Wang Haili, Wu Jiuwei, Lin Xuejian. Lin Xuejian's experience in using scalp acupuncture to treat some brain-derived diseases[J]. Chinese Acupuncture and Moxibustion, 2005, 25(10): 729-732

[17] Zhao Yong, Jin Bingxu, Liu Zhenhuan. Clinical observation on Lin's scalp acupuncture treatment of children with autism[J]. Shanghai Journal of Acupuncture, 2015, 34(08): 754-757.

[18] Wu Jiuwei, Wang Haili, Lin Xuejian. Clinical observation on the treatment of insomnia with five needles forehead $[\mathrm{J}]$. Journal of Traditional Chinese Medicine Literature, 2005(3): 48-49.

[19] Wang Minji, Zhu Jiang, Yang Yongqing. The complete book of Chinese acupuncture and moxibustion[M]. Henan Science and Technology Press, 2012.

[20] Liang Fanrong, Fang Jianqiao, Hu Ling, et al. Acupuncture. 2nd edition[M]. Shanghai: Shanghai Science and Technology Press, 2012.

[21] Wang Haili. Preliminary study on the application of pressure detection method in Lin's scalp acupuncture[J]. 
Chinese Acupuncture and Moxibustion, 2011, 31(05): 432-434.

[22] Tang Jiali, Wu Xiaolan, Zhu Zhengming, etc. Clinical observation on 50 cases of anxiety-induced insomnia treated by scalp needle extraction[J]. Zhejiang Journal of Traditional Chinese Medicine, 2016, 51(9): 668-669

[23] Wang Wei, Zhou Guobin, Yu Zhishun. Chief physician $\mathrm{Yu}$ Zhishun's experience in scalp acupuncture treatment $[\mathrm{J}]$. Modern Distance Education of Chinese Medicine, 2003, 1(6): 28.

[24] Li Wen, Li Shaomin, Xu Xiangyang, et al. The effect of exercise therapy combined with Tang's scalp acupuncture on the daily life of patients with early stroke hemiplegia[J]. Ningxia Medical Journal, 2012, 34(10): 981-982.

[25] Zhang Zidi, Wang Ruiqing, Liu Jingxuan, et al. Comparison and analysis of different schools of scalp acupuncture[J]. Acupuncture Research, 2021, 46(09): 809-814.

[26] Xu Chunhua, Fan Gangqi, Zhao Yang. Comparison and application of scalp acupuncture schools[J]. Chinese Acupuncture and Moxibustion, 2016, 36(06): 663-667.

[27] Li Qian, Huang Yinghua, Liu Yaolun, et al. The effect of acupuncture on sleep time and the content of serotonin and dopamine in the striatum of rats with stress gastric mucosal injury[J]. Chinese Journal of Information on Traditional Chinese Medicine, 2015, 22(5): 48 -51.

[28] Ren Sheng, Bao Chunling. Analyze the clinical application and mechanism of scalp acupuncture from various points distribution[J]. Shanghai Journal of Acupuncture and Moxibustion, 2018, 37(5): 575-582

[29] Wang Qiong, Xing Haijiao, Bao Na, et al. Research on the clinical application characteristics of scalp acupuncture based on data mining[J]. Acupuncture Research, 2018, 43(03): 199-203.

[30] Wan Qingyun, Wu Wenzhong, Liu Chengyong, et al. Research progress in scalp acupuncture treatment of insomnia[J]. Sichuan Traditional Chinese Medicine, 2018, 36(04): 201-204. 